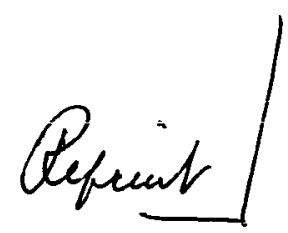

\title{
Flutter Analysis of Flat Rectangular Panels Based on Three-Dimensional Supersonic Potential Flow
}

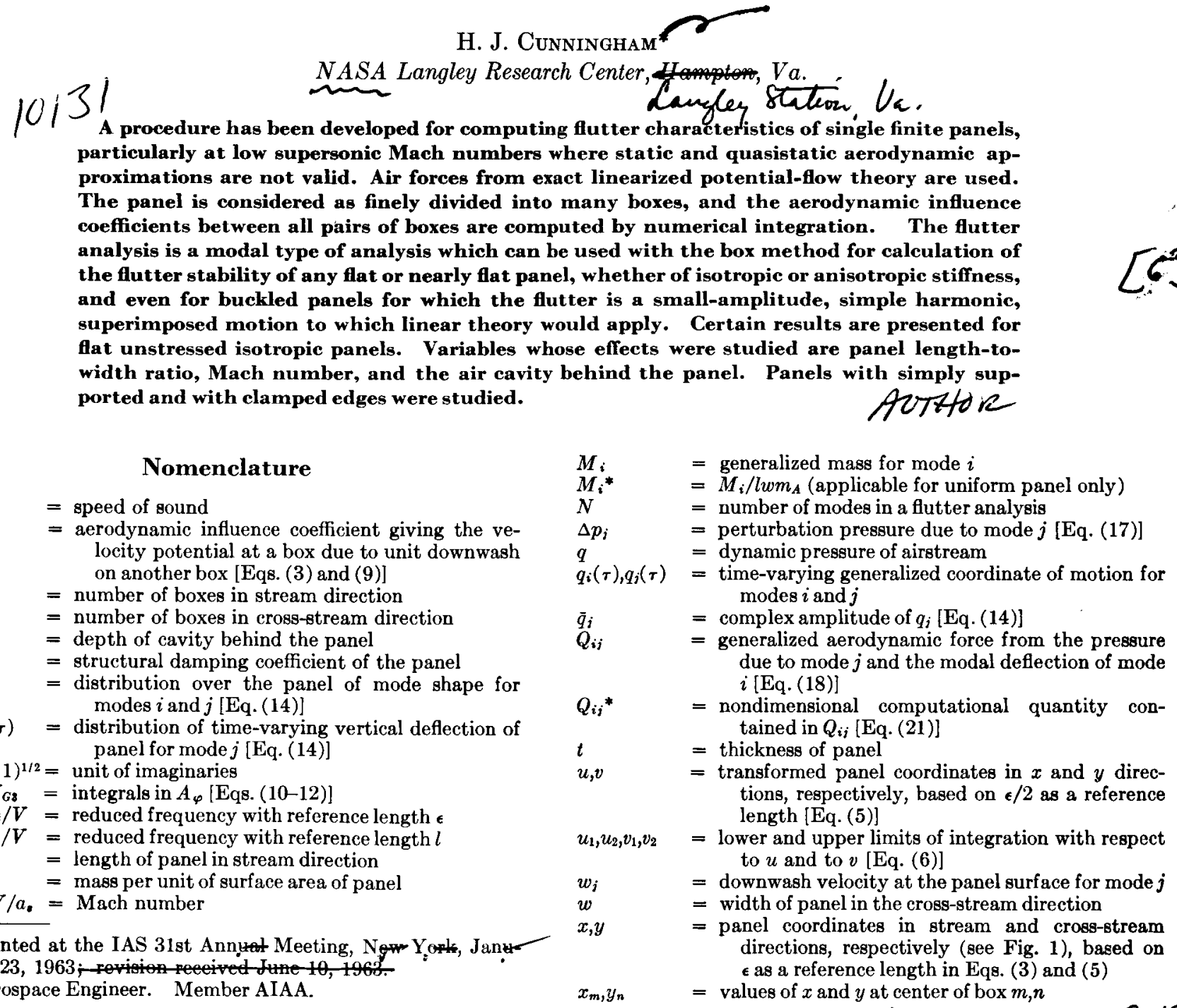

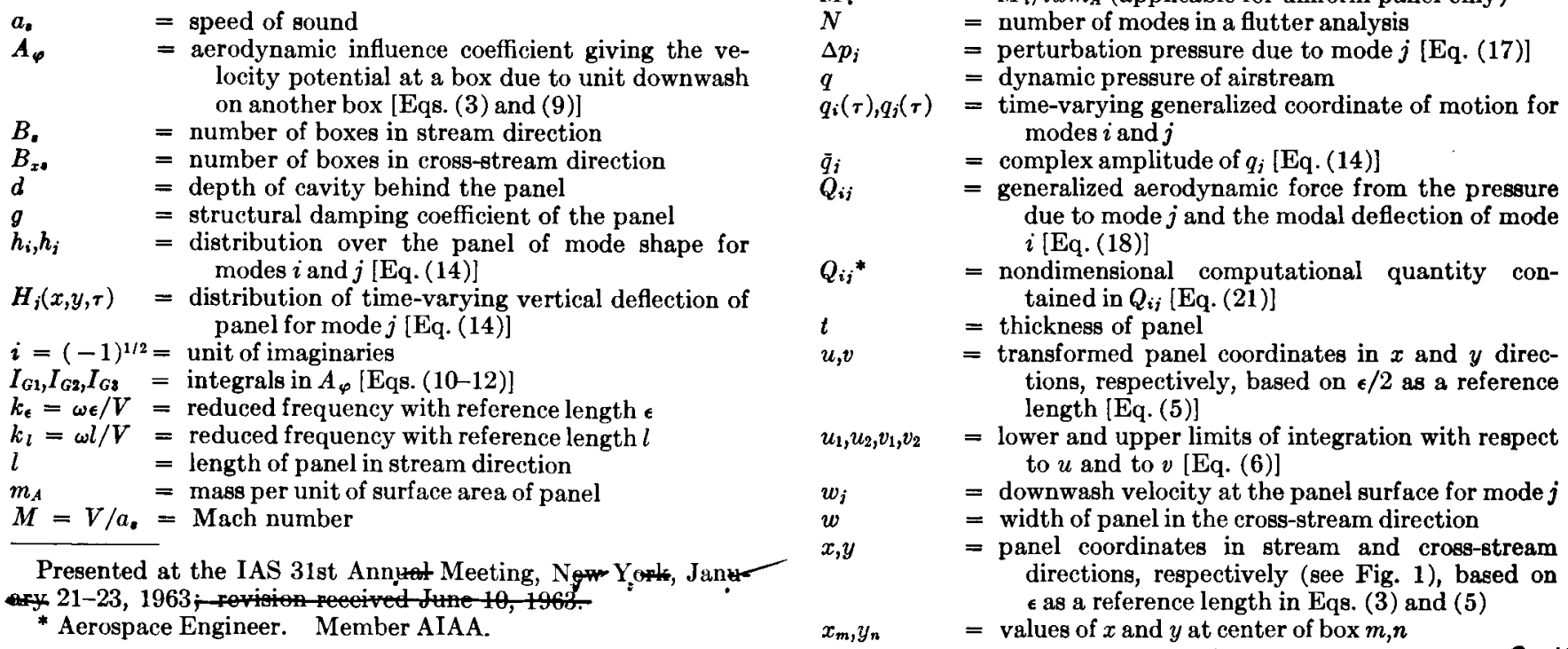




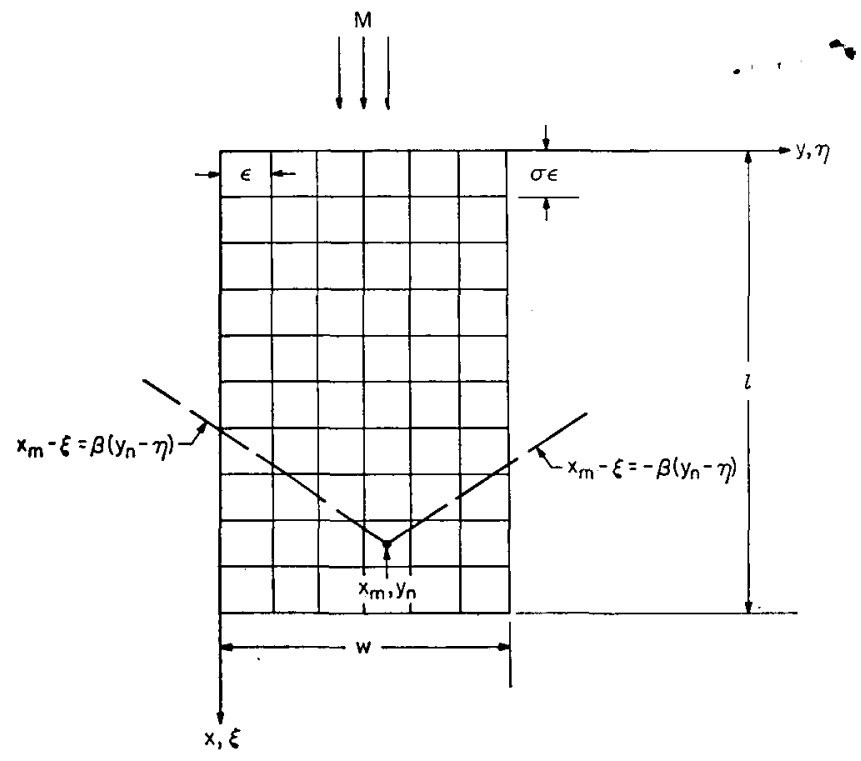

Fig. 1 Plan view of panel divided into boxes with coordinate system, dimensions, and a forward-facing Mach cone with apex at box center $x_{m}, y_{n}$.

$$
\begin{aligned}
& \bar{x}, \bar{y}=\text { panel coordinates based on reference lengths } l \\
& \text { and } w \text {, respectively } \\
& \alpha, \alpha^{*} \quad=\text { see Eqs. }(26-28) \\
& \beta \quad=\left(M^{2}-1\right)^{1 / 2} \\
& \epsilon=w / B_{x s}=\text { width of box } \\
& \eta \quad=\text { dummy variable of integration for } y \\
& \mu=m_{A} / \rho l=\text { panel-to-air mass density ratio } \\
& \xi \quad=\text { dummy variable of integration for } x \\
& \rho=\text { density of airstream } \\
& \sigma \quad=l B_{x s} / w B_{a} \text {, length-to-width ratio of box } \\
& \tau \quad=\text { time } \\
& \varphi=\varphi(m, n)=\text { velocity potential at center of box } m, n \\
& \bar{\varphi}(m, n)=\text { velocity potential at center of box } m, n \text { due to unit } \\
& \omega \quad=\text { frequency of flutter motion } \\
& \omega_{i}, \omega_{j}, \omega_{B}=\text { natural frequencies of panel modes } i \text { and } j \text { and of } \\
& \Omega=\left(\omega_{B} / \omega\right)^{2}(1+i g) \text {, flutter eigenvalue parameter } \\
& \bar{\Omega} \quad=M^{2} k_{\epsilon} / \beta^{2}
\end{aligned}
$$

\section{Subscripts}

\section{Introduction}

$\mathbf{T}$ HERE has been considerable interest and activity in the analysis of panel flutter. Perhaps the most recent reviews of the work done are those by Stocker ${ }^{1}$ and by Fung ${ }^{2}$ which cover both theoretical and experimental investigations. The present paper is concerned with an important type of panel and speed regime for which analysis has been lacking. That type of panel is the single rectangular one (embedded in a rigid surface), and the speed regime is the low supersonic, especially below Mach number $2^{1 / 2}$. For these Mach numbers, simple approximations for the aerodynamic forces, such as from Ackeret theory or piston theory, are not satisfactory. Use of the exact theory for linearized supersonic flow is a logical step. This exact theory was applied by Luke and St. John ${ }^{3}$ to an effectively infinitewidth panel separated into bays by supports equally spaced along the width. Zeijdel ${ }^{4}$ obtained additional results for such a panel with added supports that divide the length of the panel into equal bays. For this type of panel the generalized aerodynamic forces needed in the flutter determinant can be obtained by evaluation of closed-form expressions. For finite-width panels, however, closed-form expressions have not been found for the aerodynamic force terms even for simple mode shapes of the panel.

The subject of the present paper is the determination of the aerodynamic forces by, in effect, a numerical integration process and the subsequent use of the air forces to compute flutter boundaries. For this problem, current high-speed computing machines make practicable the obtaining of solutions by such "brute force" methods that would not be attempted otherwise. The panel is considered to be finely divided into a large number of equal-size boxes. The aerodynamic influence coefficient of each box on each other box is calculated and used in a modal-type flutter stability analysis. The mode shape properties can be supplied from either calculation or experimental measurement.

A number of flutter results are presented for flat unstressed panels with isotropic stiffness and by use of calculated mode shapes. Calculated modal frequencies were used, with one exception. The principal variables studied are the panel length-to-width ratio and the flow Mach number. Effects of the air cavity behind the panels also are examined in a qualitative sense.

Although the results presented are limited to simple panels, this modal analysis with a box method has a broad application and lends itself to the calculation of the flutter stability of any flat or nearly flat panel, whether unstressed or stressed (as by thermal expansion), whether of isotropic or anisotropic stiffness, and even for buckled panels for which the flutter is a small-amplitude, simple harmonic, superimposed motion to which linear theory would apply.

\section{Analysis}

The panel to be analyzed and the coordinate system are shown in Fig. 1. It is a single rectangular pancl of length $l$, width $w$, and the side edges are aligned with the remote wind direction. It is a flexible part of an otherwise inflexible surface which extends at least far enough so that upper and lower surfaces of the panel induce no aerodynamic effects upon cach other. The supersonic stream passes over the upper surface. No account is taken of any perturbation pressures on the panel lower surface except for some remarks regarding cavity effects near the end of the paper.

The panel is divided into a number of equal-size rectangular boxes. The number of boxes in the stream and crossstream direction are $B_{s}$ and $B_{x s}$, respectively. For convenience and for purposes of reference in the computational procedure, the boxes are numbered in sequence, beginning at the box nearest the origin of coordinates. In the stream direction the index $m=0,1,2, \ldots B_{s}-1$, and in the crossstream direction $n=0,1,2, \ldots B_{x s}-1$. A second set of box index numbers is needed, and these are $\mu=0,1,2, \ldots$ $B_{s}-1$ and $\nu=0,1,2, \ldots B_{x s}-1$. Thus, the aerodynamic influence upon any box $m, n$ due to any other box $\mu, \nu$ can be referred to.

\section{Aerodynamic Influence Coeflicients}

It is assumed that the panel is divided into a sufficiently fine gridwork of boxes so that the downwash over any one box can be taken as uniformly distributed at any instant and that the resulting pressure perturbation at the center of each box is a sufficiently accurate average of the pressure distribution over that box.

For convenience in the computational procedure, a reference length is chosen as $\epsilon$, the width of a box; that is, $\epsilon=$ $w / B_{x s}$. The velocity potential at the center of box $m, n$ due 
to a uniformly distributed downwash $w(\mu, \nu)$ over the box $\mu, \nu$ can be expressed for a simple-harmonic time variation as

$$
\bar{\varphi}(m, n)=\epsilon w(\mu, \nu) A_{\varphi}(r, s)
$$

where

$$
r=m-\mu \quad s=n-\nu
$$

both sides of the Mach cone). For this condition

$$
I_{G 1}=\frac{1}{2} \int_{u_{1}}^{u_{2}} e^{-(\bar{\beta} / 2) u} J_{0}\left(\frac{\beta \bar{\Omega}}{2 M} u\right) d u
$$

The second form of Eq. (9) occurs for portions of a box cut by one side of the Mach cone $(\nu \neq n)$, so that the limit $v_{2}=$

and, from Eq. (34) of Ref. 5,

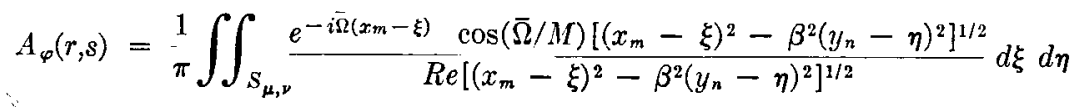

in which the area of integration $S_{\mu, \nu}$ is the area of box $\mu, \nu$. In the denominator, the indicated real part of the radical term has a nonzero value for any part of $S_{\mu, \nu}$ which lies ahead of the forward facing Mach cone with apex at $x_{m}, y_{n}$ (see Fig. 1). All boxes and parts of boxes lying behind the Mach cone have a zero contribution to $A_{\varphi}(r, s)$. The frequency Mach number parameter $\bar{\Omega}$ is

$$
\bar{\Omega}=M^{2} k_{\epsilon} / \beta^{2}
$$

where the reduced frequency $k_{\epsilon}=\omega \epsilon / V$ contains the chosen reference length $\epsilon$

As in Ref. 5, a transformation of variable is made as follows:

$$
x_{m}-\xi=\beta u / 2 \quad y_{n}-\eta=v / 2
$$

Then

$$
\begin{aligned}
A_{\varphi}(r, s)=\frac{1}{2 \pi} \int_{u_{1}}^{u_{2}} e-i(\beta \bar{\Omega} / 2) u d u \times \\
\qquad \int_{v_{1}}^{v_{2}} \frac{\cos (\beta \bar{\Omega} / 2 M)\left(u^{2}-v^{2}\right)^{1 / 2}}{\left(u^{2}-v^{2}\right)^{1 / 2}} d v
\end{aligned}
$$

where the upper and lower limits of the surface integrals, indicated by $v_{1}, v_{2}, u_{1}$, and $u_{2}$, are determined for any given box by its edges except where the forward Mach cone emanating from $x_{m}, y_{n}$ passes through the box.

The integral of Eq. (6) is the one to be evaluated numerically. It is to be noted that the integrand contains a singularity of order $\left(-\frac{1}{2}\right)$ at $u=v$, that is, along the Mach lines from $x_{m}, y_{n}$. In order to avoid the potential inaccuracies involved in integrating in the vicinity of this singularity, the numerator of the integrand is replaced as follows:

$$
\begin{array}{r}
\cos (\beta \bar{\Omega} / 2 M)\left(u^{2}-v^{2}\right)^{1 / 2} \equiv \\
{\left[\cos (\beta \bar{\Omega} / 2 M)\left(u^{2}-v^{2}\right)^{1 / 2}-1\right]+1}
\end{array}
$$

The integration with respect to $v$ can be partly carried out formally:

$$
\begin{aligned}
& \int_{v_{1}}^{v_{2}}\left(\frac{1}{\left(u^{2}-v^{2}\right)^{1 / 2}}+\frac{\cos (\beta \bar{\Omega} / 2 M)\left(u^{2}-v^{2}\right)^{1 / 2}-1}{\left(u^{2}-v^{2}\right)^{1 / 2}}\right) d v= \\
& \cos ^{-1} \frac{v_{1}}{u}-\cos ^{-1} \frac{v_{2}}{u}+\int_{v_{1}}^{v_{2}} \frac{\cos (\beta \bar{\Omega} / 2 M)\left(u^{2}-v^{2}\right)^{1 / 2}-1}{\left(u^{2}-v^{2}\right)^{1 / 2}} d v
\end{aligned}
$$

The integrand of the remaining integral with respect to $v$ is zero at $u=v$, and the integral can be evaluated more accurately. Substitution of Eq. (8) into $A_{4}(r, s)$ gives the result

$$
\begin{array}{r}
A_{\varphi}(r, s)=\frac{1}{2 \pi} \int_{u_{1}}^{u_{2}} e^{-i(\beta \bar{\Omega} / 2) u}\left(\cos ^{-1} \frac{v_{1}}{u}-\cos ^{-1} \frac{v_{2}}{u}+\right. \\
\left.\int_{v_{1}}^{v_{2}} \frac{\cos (\beta \bar{\Omega} / 2 M)\left(u^{2}-v^{2}\right)^{1 / 2}-1}{\left(u^{2}-v^{2}\right)^{1 / 2}} d v\right) d u
\end{array}
$$

There is a need for only three forms of Eq. (9). The first is for the condition that $v_{1}=-v_{2}=-u$ (which can occur only for $\nu=n$, and then only for the portion of a box $\mu, \nu$ cut by

$$
\begin{aligned}
I_{G 2}= & \frac{1}{2 \pi} \int_{u_{1}}^{u_{2}} e^{-i(\beta \bar{\Omega} / 2) u}\left(\cos ^{-1} \frac{v_{1}}{u}+\right. \\
& \left.\int_{v_{1}}^{u} \frac{\cos (\beta \bar{\Omega} / 2 M)\left(u^{2}-v^{2}\right)^{1 / 2}-1}{\left(u^{2}-v^{2}\right)^{1 / 2}} d v\right) d u
\end{aligned}
$$

The third form $I_{G 3}$ occurs for boxes that are completely ahead of the Mach cone and also for portions of boxes ahead of the point where the Mach cone passes out through the side of the box:

$$
\begin{aligned}
I_{G 3}=\frac{1}{2 \pi} \int_{u_{1}}^{u_{2}} e^{-(\beta \bar{\Omega} / 2) u}\left(\cos ^{-1} \frac{v_{1}}{u}-\cos ^{-1} \frac{v_{2}}{u}+\right. \\
\\
\left.\int_{v_{1}}^{v_{2}} \frac{\cos (\beta \bar{\Omega} / 2 M)\left(u^{2}-v^{2}\right)^{1 / 2}-1}{\left(u^{2}-v^{2}\right)^{1 / 2}} d v\right) d u
\end{aligned}
$$

Table 1 shows all possible relative locations of a box and the Mach cone. The applicable integral, whether $I_{G 1}, I_{G 2}$, or $I_{G 3}$ is shown, and the limits of integration $v_{1}, v_{2}, u_{1}$, and $u_{2}$ are listed. The limits shown are consistent with the reference length $\epsilon$ chosen for Eq. (1) and with the transformation of Eq. (5). The quantity $\sigma=l B_{x s} / w B_{a}$ is the length-to-width ratio of a box.

With the assumptions that have been made, the aerodynamic influence coefficients have right-left symmetry about $s=0$; that is,

$$
A_{\varphi}(r, s)=A_{\varphi}(r,-s)
$$

Thus, all of the $A_{\varphi}(r, s)$ which are needed for any box are obtained for either one of the rear corner boxes.

Once all $A_{\varphi}(r, s)$ are computed, the total $\varphi_{j}(m, n)$ for any downwash mode $j$ at the center of any single box $m, n$ is obtained by a matrix multiplication:

$$
\begin{aligned}
\varphi_{j}(m, n) & =V \epsilon \sum_{\mu} \sum_{\nu} \frac{w_{j}(\mu, \nu)}{V} A_{\varphi}(r, s) \\
& =V \epsilon\left\lfloor\frac{w_{j}(\mu, \nu)}{V}\right\rfloor\left\{A_{\varphi}(r, s)\right\}
\end{aligned}
$$

in which any element $A_{\varphi}$ is zero where $m-\mu=r$ would be negative.

\section{Downwash}

The dovnwash $w_{j}$ is employed as follows. Let the panel deflection in mode $j$ be expressed for simple-harmonic time variation as

$$
H_{i}(x, y, \tau)=q_{j}(\tau) h_{i}(x, y)=\bar{q}_{i}(\tau) e^{i \omega \tau} h_{i}(x, y)
$$

where $\bar{q}_{j}$ is the complex amplitude of the generalized coordinate of motion, and $h_{j}(x, y)$ is the shape of the deflection mode. The downwash ratio is

$$
\begin{aligned}
\frac{w_{j}}{V} & =\frac{\partial H_{j}}{\partial(l \bar{x})}+\frac{1}{V} \frac{\partial H_{j}}{\partial \tau} \\
& =\frac{q_{j}}{l}\left(\frac{\partial h_{j}}{\partial \bar{x}}+i \frac{\omega l}{V} h_{j}\right)
\end{aligned}
$$


where $\bar{x}$ is a convenient streamwise coordinate that is zero at the panel leading edge and 1.0 at the trailing edge. Substitution of Eq. (16) into Eq. (14) gives the result

$$
\varphi_{j}(m, n)=V \epsilon \frac{q_{i}}{l}\left\lfloor\frac{\partial h_{i}}{\partial \bar{x}}+i \frac{\omega l}{V} h_{j}\right\rfloor\left\{A_{\varphi}(r, s)\right\}
$$

in which $h_{i}$ is $h_{j}\left(x_{\mu}, y_{\nu}\right)$. Thus, the $\varphi_{i}(m, n)$ are obtainable in a systematic manner for each mode $j$ used in a flutter analysis.

\section{Generalized Aerodynamic Forces}

The perturbation pressure $\Delta p_{i}$ is obtained from the velocity potential by

$$
\begin{aligned}
\Delta p_{i} & =-\rho\left[V \frac{\partial \varphi_{i}}{\partial(l \bar{x})}+\frac{\partial \varphi_{j}}{\partial \tau}\right] \\
& =-\frac{\rho V}{i}\left(\frac{\partial \varphi_{i}}{\partial \bar{x}}+i \frac{\omega l}{V} \varphi_{i}\right)
\end{aligned}
$$

The generalized aerodynamic force term $Q_{i j}$ needed in the

Table 1 Types of integrals and limits of integration for computing $A_{\varphi}(r, s)$ for all possible relative locations of the box $\mu, \nu$ and the Mach cone from box $m, n$

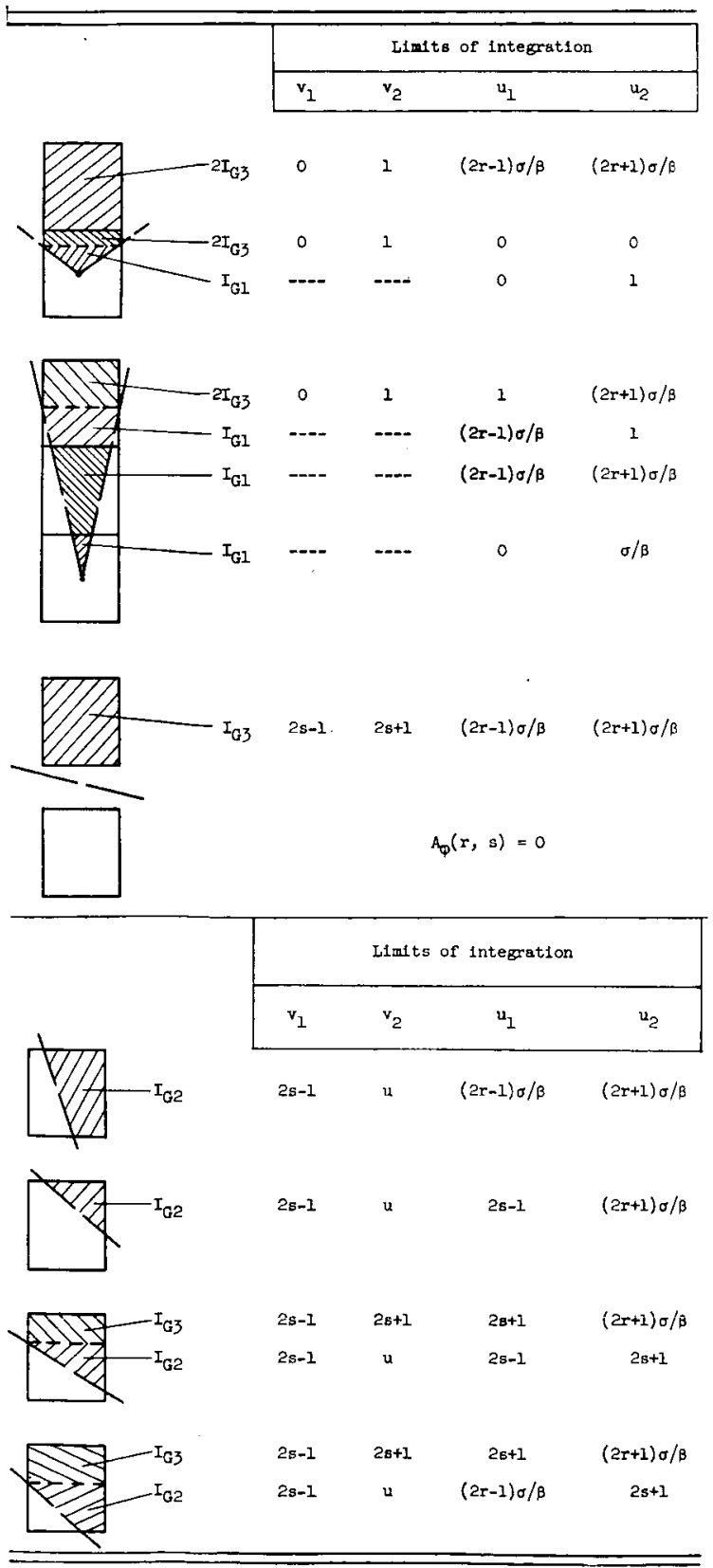

flutter determinant is the integral over the panel surface of the product of the deflection mode shape $h_{i}$ times $\Delta p_{i}$; that is,

$$
Q_{i i}=-\frac{\rho V}{l} \iint h_{i}\left(\frac{\partial \varphi_{j}}{\partial \bar{x}}+i \frac{\omega l}{V} \varphi_{j}\right) d(l \bar{x}) d(w \bar{y})
$$

The coordinate $\bar{y}$ ranges from zero at the left edge to 1.0 at the right edge. If the term $h_{i}\left(\partial \varphi_{i} / \partial \bar{x}\right)$ is integrated by parts, there is obtained

$$
\begin{aligned}
Q_{i i}=-\frac{\rho V}{l} \int\left[h_{i}\left(x_{t e}, y\right) \varphi_{j}\left(x_{t e}, y\right)-\right. \\
\left.\qquad \varphi_{i}\left(\frac{\partial h_{i}}{\partial \bar{x}}-i \frac{\omega l}{V} h_{i}\right) d(l \bar{x})\right] d(w \bar{x})
\end{aligned}
$$

Since $h_{i}\left(x_{t e}, y\right)$ is zero for panels supported at the trailing edge,

$$
Q_{i j}=\frac{\rho V}{l} \iint \varphi_{i}\left(\frac{\partial h_{i}}{\partial \bar{x}}-i \frac{\omega l}{V} h_{i}\right) d(l \bar{x}) d(w \bar{y})
$$

In Eq. (21) the quantity [ $\left.\left(\partial h_{i} / \partial \tilde{x}\right)-i(\omega l / V) h_{i}\right]$ readily is seen to be the complex conjugate of the amplitude of the downwash ratio for mode $i$ [where the subscript $i$ is not to be confused with the unit of imaginaries $i=(-1)^{1 / 2}$.

In practice the integral of Eq. (21) is evaluated by a summation or matrix multiplication that can be put in the convenient form

$$
Q_{i j}=\rho V^{2} \frac{w^{2}}{B_{0}\left(B_{x \iota}\right)^{2}} \frac{q_{i}}{l} Q_{i j}^{*}
$$

where

$$
Q_{i j}{ }^{*}=\left[\frac{\partial h_{i}}{\partial \bar{x}}-i \frac{\omega l}{V} h_{i}\right]\left\{\left[\frac{\partial h_{i}}{\partial \bar{x}}+i \frac{\omega l}{V} h_{j}\right]\left\{A_{\varphi}\right\}\right\}
$$

\section{Solution of the Flutter Determinant}

For the present purpose it will be assumed that the modes to be used in the flutter solution are orthogonal, that is, with neither inertia nor elastic coupling between modes. The equation expressing the dynamic equilibrium of motion, derived from application of the Galerkin method, is

$$
\left[\omega^{2}-\omega_{i}^{2}(1+i g)\right] M_{i} q_{i}+\sum_{j} Q_{i j}=0
$$

$$
(i=1,2, \ldots N)
$$

where $\omega_{i}$ is the natural frequency of mode $i, N$ is the number of modes used in the analysis, $g$ is an assumed damping coefficient used as a flutter indicator, and $M_{i}$ is the generalized mass of mode $i$ :

$$
M_{i}=l w \int_{0}^{1} \int_{0}^{1} m_{A}(x, y)\left[h_{i}(x, y)\right]^{2} d \bar{x} d \bar{y}
$$

with $m_{A}$ representing the panel mass per unit area.

With use of the definition $\Omega=\left(\omega_{B} / \omega\right)^{2}(1+i g)$ as the flutter eigenvalue parameter, where $\omega_{B}$ is any chosen base or reference frequency, Eq. (24) can be rearranged in a form convenient for eigensolution:

$$
\left[-\Omega+\left(\frac{\omega_{B}}{\omega_{i}}\right)^{2}\right] q_{i}+\left(\frac{\omega_{B}}{\omega_{i}}\right)^{2} \frac{\alpha}{k_{l}^{2} M_{i}} \sum_{j} q_{j} Q_{i j}^{*}=0
$$

where

$$
\alpha=\rho \frac{l}{B_{e}}\left(\frac{w}{B_{x \bullet}}\right)^{2}
$$

and $k_{l}=\omega l / V$ is the reduced frequency based on the reference length $l$. For panels with a uniform mass distribution $m_{A}$, the quantity $\alpha / M_{i}$ in $\mathrm{Eq}$. (26) can be replaced as follows:

$$
\alpha / M_{i}=\left(\alpha^{*} / M_{i}^{*}\right)(1 / \mu)
$$


where

$$
\begin{aligned}
& \alpha^{*}=w / l B_{0} B_{x i^{2}} \\
& M_{i}{ }^{*}=\int_{0}^{1} \int_{0}^{1} h_{i}{ }^{2} d \bar{x} d \bar{y}
\end{aligned}
$$

and $1 / \mu=\rho l / m_{A}$ is the air-to-panel mass ratio. Thus, one now has all the necessary quantities for computing flutter boundaries. Panel dimensions and natural frequencies are employed only as ratios. The downwash ratios and other parameters involving the mode shapes are in forms that are independent of the panel size.

As is commonly the case with exact air forces, the flutter boundary cannot be computed directly, and an indirect method of computing and cross-plotting is necessary. In advance of a flutter solution for any given panel and Mach number, choices are made for $B_{s}, B_{x v}$, and the number of modes in the analysis. The downwash quantities $\partial h_{i} / \partial \bar{x}$ and $h_{i}$ are computed or obtained from experiment at the center of each box for each mode $i$ (or $j$ ) and arranged systematically for use in the matrix multiplications. The quantities $M_{i}$ and $\alpha$ (or $M_{i}^{*}, 1 / \mu$, and $\alpha^{*}$ for panels with uniform $m_{A}$ ) are computed, except that allowance for varying the air density in $\alpha$ (or $1 / \mu)$ is retained.

Following judicious choice of the reduced frequency $k_{l}$, the block of values of $A_{\varphi}(r, s)$, then the $\varphi_{j}(m, n)$ at each box center for each mode, and then the generalized aerodynamic forces $Q_{i j}$ are computed and employed in the flutter determinant. For each of a number of values of the air density, the complex eigenvalues $\Omega$ are computed. There is one - eigenvalue corresponding to each chosen panel mode. By plotting curves of $g$ against $1 / \mu$ for a sufficient range of air densities, the existence or nonexistence of an eigenvalue with $g=\mathbf{0}$ for some air density is established for each mode. An associated curve of the stiffness parameter $\omega_{1} l / V$ or $\omega_{1} l / a$. is plotted against mass ratio-one curve for each eigenvalue. (The use of the speed of sound $a$, rather than $V$ permits direct comparison of flutter boundaries for various Mach numbers.)

On a plot of $1 / \mu$ against stiffness parameter, points now can be placed which represent $g=0$ or some other small value of $g$ believed representative of the actual hysteresis structural damping of the panel. Each point is on a separate stability boundary.

By successive judicious choices of $k_{l}$, a series of points can be placed on the plot of $1 / \mu$ against stiffness parameter and stability boundaries drawn through the points. Four different types of stability boundaries have been found, in general, and three of them are illustrated in Fig. 2. For each type, a boundary for $g=0$ and one for $g=0.01$ (as illustrative of some small value) are shown, and the arrows point in the direction of increasing reduced frequency $k_{l}$. The type of boundary of Fig. 2a predicts that, if $g$ were zero, a fixed panel thickness would be needed as the air density tends toward zero, but if $g$ is not zero, panel thickness goes toward zero as air density does.

For the type of boundary of Fig. 2b, the unstable region shrinks with increasing $g$ and vanishes for some value that may be extremely small or as large as $g=0.05$ or possibly larger, depending upon the panel and flow parameters. The crossing point of the boundary for $g=0$ in both Figs. 2a and $2 \mathrm{~b}$ through $1 / \mu=0$ occurs at a value of $k_{l}$ for which the imaginary part of $Q_{i i}\left(k_{l}\right)$ passes through zero. For the type of boundary of Fig. 2c, small increases of $g$ have little or no effect, and this small effect can be either stabilizing or destabilizing, depending on the particular panel and flow parameters. An important point regarding the boundary of Fig. $2 \mathrm{c}$, as well as the dashed boundary of Fig. 2a, concerns their resemblance to a parabola. For any boundary or portion of a boundary which is a parabola accurately described by $1 / \mu=\left(\omega_{1} l / a_{s}\right)^{2}$ times a constant, the air density and the panel thickness ratio $t / l$ to prevent flutter are related by the formula $t / l(\rho)^{1 / 3}=$ const. Such a relationship is contained
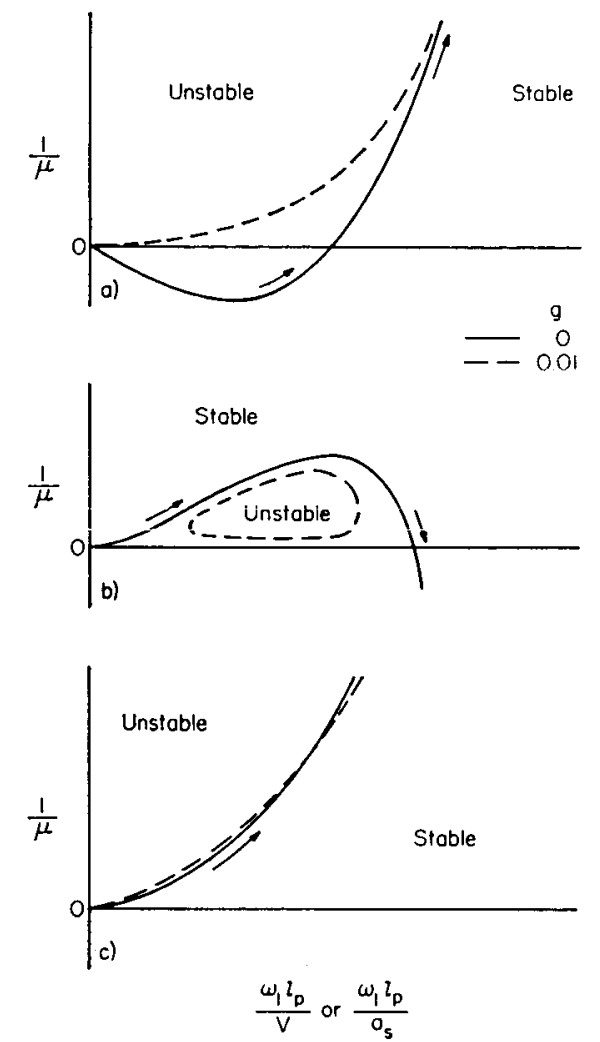

Fig. 2 Three general types of stability boundaries for $g=$ 0 and for $g=0.01$. Arrows indicate direction of increasing reduced frequency $\boldsymbol{k}_{7}$.

in the panel flutter parameter $(E \beta / q)^{1 / 3}(t / l)$, which has been evolved and used by several investigators. The fourth possible type of boundary found from a flutter determinant is one that falls entirely in the negative $1 / \mu$ region, with the positive $1 / \mu$ region being stable with respect to it.

For any given panel and flow parameters, a set of stability boundaries is computed according to the number of modes used in the analysis. The flutter boundary separates the region that is stable with respect to all stability boundaries from the region that is unstable with respect to at least one stability boundary. It is the duty of the investigator to establish that the flutter boundary is converged, that is, that enough modes have been used in the analysis so that additional modes do not alter the flutter boundary in any im. portant respect.

\section{Results and Discussion}

\section{Effect of Panel Length-to-Width Ratio on Flutter}

Results have been calculated for several panel length-towidth ratios in the range from $l / w=0$ (the two-dimensional panel) to 10 , for $M=1.3$, and with $g=0$. The panel edges were either clamped or simply supported (pinned). The results for an aluminum panel at sea level are shown on Fig. 3 . The abscissa is the length-to-width ratio, and the ordinate is a panel flutter parameter $(\beta E / q)^{1 / 3}(t / l)$. Analytical results calculated for $M=1.3$ and with $g=0$ are shown for panels with clamped edges by the square points connected by the long-dash line and for panels with simply supported (pinned) edges by the round points connected by the shortdash line. Also shown for comparison on Fig. 3 is the experimental flutter envelope from NASA TN D-4516 which was obtained from tests on many panels with different sizes and materials, for Mach numbers from 1.6 to 6, at different temperatures, and with various compressive loadings and amounts of buckling. The analytical results fall below the experimental envelope as would be expected, since the analysis 


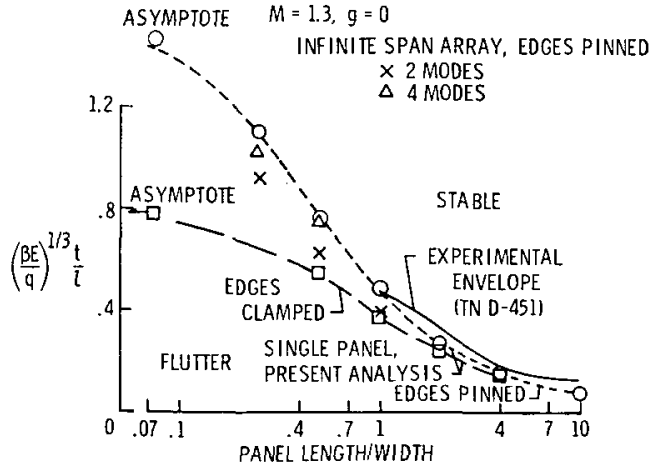

Fig. 3 Analytical variation of panel flutter parameter with $\boldsymbol{l} / \boldsymbol{w}$ for aluminum panels at sea level compared with the experimental envelope of Ref. 6 . Present results for a single panel and previous results for an infinite span array of panels are shown for $M=1.3$ and $g=0$.

is of flat unstressed panels, and the experimental envelope is established largely by marginally buckled panels. Also shown as a matter of interest are a few results from Refs. 3 and 4 for an infinite span array of panel bays.

A point of interest concerns the flutter frequency and flutter mode. For all the length-width ratios at least up through 4 on Fig. 3, the flutter frequency was near and usually slightly above the first-natural-mode frequency. But even where the flutter frequency was almost exactly equal to the first natural frequency, the flutter mode was a coupling of more than one natural mode. The degree of coupling was small for the low values of $l / w$ but increased with increasing $l / w$. For $l / w=10$, there was a great change in the flutter mode. Twelve modes were required in order to obtain a converged flutter boundary. The flutter frequency was between the eighth and ninth natural frequencies. The largest component present in the flutter mode was the ninth natural mode, and the proportions of modes near to the ninth were also large.

\section{Effect of Mach Number}

The thickness ratio $t / l$ and the panel flutter parameter have been computed for an aluminum panel at sea level with $l / w=2$, edges clamped, and for several Mach numbers from 1.1 to 2.0. The results are given in Table 2. The thickness ratio to prevent flutter is virtually constant for $M$ of 1.2 to 1.5 , is $5 \%$ higher at $M=2.0$, and is $6 \%$ lower at $M=1.1$. This trend for low supersonic Mach numbers is in sharp contrast to the result for two-dimensional panels for which a great increase in thickness ratio is predicted to be required for Mach numbers less than about $2^{1 / 2}$. The ratios of flutter frequency to first natural frequency fell between 1.0 and 1.1 for this panel and Mach number range.

Reference 7 (Lock and Fung) reports the results of panel flutter experiments at low supersonic Mach numbers from 1.16 to 1.45 . The panels tested were square or nearly so $(l / w=0.95,1.00$, and 1.06$)$, were clamped at their leading edges, approximately simply supported at their trailing edges, and free on their side edges to allow the flutter motion to

Table 2 Clamped-edge aluminum panels at sea level

\begin{tabular}{ccc}
\hline$M$ & $t / l$ & $(\beta E / q)^{1 / 3}(t / l)$ \\
\hline 1.1 & 0.00292 & 0.212 \\
1.2 & 0.00311 & 0.241 \\
1.3 & 0.00312 & 0.251 \\
1.4 & 0.00311 & 0.248 \\
1.5 & 0.00311 & 0.247 \\
2.0 & 0.00327 & 0.248 \\
\hline
\end{tabular}

be "two-dimensional." In Fig. 21 of Ref. 7, the experimental thickness ratio to prevent flutter is shown as a function of Mach number for a condition of no pressure difference through the panel. The increase of thickness as $M$ decreases below $2^{1 / 2}$ was found to be much smaller than predicted by analysis of a two-dimensional panel. Even though the panel motion showed no cross-stream variation, it seems evident that the aerodynamic forces exerted on the panel are not truly two-dimensional, especially near the panel side edges. The reason is that from the panel side edge to the tunnel side wall there is an area of nonmoving tunnel surface of width equal to about $6.2 \%$ of the panel width. If the tunnel side wall acted as a reflector, the image of the panel beyond the side wall would be at a distance of $12.4 \%$ of pancl width from the pancl.

Since the images could not be accounted for with the present analysis, a single square panel was analyzed as a matter of interest. Six modes were used for the panel clamped at leading edge, simply supported at trailing edge, and free at side edges. The measured frequencies for the first two modes (the only ones available) and calculated frequencies for modes 3 to 6 were used. Flutter calculations were made at three Mach numbers: $1.16,1.20$, and 1.30 . The results are shown on Fig. 4 for comparison with the experimental results from Ref. 7. The upper curve is for $g=0$, the next lower one for $g=0.01$, and below that one for $g=$ 0.015 . Thus, in terms of thickness ratio the analytical results with a small value of $g$ agree rather well with the experiments. However, there is a significant difference in the flutter frequency. The experimental frequency was below the first natural frequency and within about $15 \%$ of it. The analytical frequency for a single panel was slightly below the second natural frequency for $M=1.16$ and 1.20 and was slightly below the third natural frequency for $M=1.30$. Obviously, some factor or factors are not accounted for in the analysis to match the experiments, whether it is the boundary layer over the panel (as suggested in Ref. 7), the reflected images of the panel through the boundary layer on the tunnel side walls (see sketch in Fig. 4), the air in the cavity behind the panel, or the air puffing in and out of the cracks along the panel side edges.

\section{Effect of Air Cavity behind the Panel}

Panels on aircraft and missiles generally have some kind of a space behind them which can have an effect on the flutter stability of the panel. Such an effect was reported in NASA TN D- $827^{8}$ on the flutter of a Fiberglas sandwich panel with a plastic-foam core. The panel had a length of 33.38 in. and a width of 20.31 in. Most of the tests were made with cavity depth of $1.5 \mathrm{in}$. behind the panel, and flutter was obtained over a Mach number range from 1.76 to 2.87 . When the cavity was filled with layers of plywood so that the back surface of the panel touched the plywood, no flutter was obtained even though the airstream dynamic pressure was doubled. But when the cavity depth was made 0.5 in., flutter occurred at a dynamic pressure $40 \%$ lower than with the 1.5-in. cavity depth.

Since the flutter frequency was much lower than the lowest acoustic-resonance frequency of the cavity, a simplified study was made on the basis that the only action of the air in the cavity is as an air spring that resists panel deflection in any mode that would compress the air but does not affect modes not involving compression. In the analysis the odd-numbered modes $(1,3,5$, etc.) involve compression, and the evennumbered modes $(2,4$, etc.) do not. The air-spring effect is largest on mode 1 , much less on mode 3 , and essentially negligible on higher modes. The analytically determined effect for a progressively decreased cavity depth primarily is to force the first-mode frequency upward toward the unaffected second-mode frequency. For sufficiently small cavity depth, the first-mode frequency could coincide with or even go above the second-mode frequency. One finding 
Fig. 4 Comparison of analytical and experimental $t / l$ as functions of $M$ for a square brass panel with clampod leading edge and simply supported trailing edge.

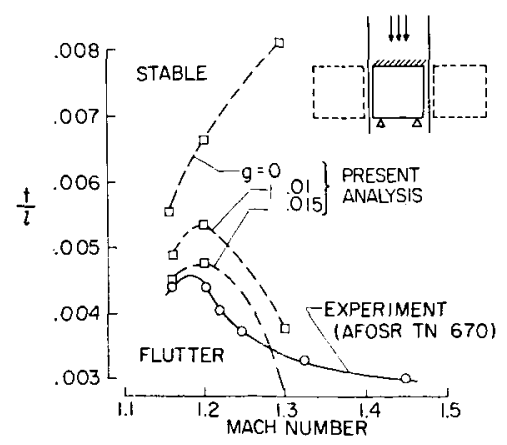

of the analysis was that determination of the natural frequencies of the panel in place over the cavity should be measured with the air density equal to (or over a range of density including) that in the cavity during the flutter experiment. This is true because the test density is often a small fraction of the density of the outside atmosphere (where the still-air panel modes and frequencies usually are measured), and because the incremental effect of the cavity air spring is proportional to the cavity air density.

Since experimental frequencies were not available from Ref. 8 for the air densities of the flutter tests, flutter results have not been computed as a function of cavity depth as a variable. Instead, the panel flutter parameter has been obtained as a function of an assumed variable first-mode frequency. The result is shown qualitatively in Fig. 5 as the panel flutter parameter vs the first-mode frequency. As the first-mode frequency increases, the flutter parameter increases at an accelerating rate, reache a maximum value in the vicinity of $\omega_{1}=\omega_{2}$, and then decreases sharply with further increase of $\omega_{1}$. It seems clear that the same trend would be obtained for decreasing cavity depth.

Finally, there are undoubtedly other effects of the cavity which would become important for sufficiently small cavity depths. These are the virtual inertia and the frictional resistance of the air being pushed back and forth in the cavity by panel modes higher than the first. There could also be acoustic resonance effects at sufficiently high frequencies. The effect of the virtual inertia of the cavity air would be to lower natural-mode frequencies. In Ref. 8 it is reported that decreasing the cavity depth from 1.5 to 0.5 in. lowered the frequencies of modes 2,3 , and 4 by appreciable amounts, but the first-mode frequency could not be found. These frequency measurements were made at essentially sea-level air density for which the cavity effects are proportionately larger than for the much lower air density of the flutter test.

\section{Concluding Remarks}

A panel flutter analysis has been developed for which the generalized aerodynamic forces are computed by the "brute force" technique of dividing the panel into a larger number of boxes and computing and employing the aerodynamic influence of each box on each other box. Such a technique can be resorted to where the acrodynamic forces are not obtainable from simple or elegant closed-form mathematical expressions. An important area of application of this technique is to finite panels at low supersonic Mach numbers. Either experimental or analytical mode shapes and frequencies can be used. Thus, this type of analysis can be applied to any flat or nearly flat panel, whether unstressed or stressed (as by thermal expansion), whether of isotropic or anisotropic stiffness, and even to a small-amplitude flutter superimposed on a buckled deflection.

Results are presented only for flat unstressed isotropic rectangular panels with side edges parallel to the air-stream direction and for a condition of no pressure difference that

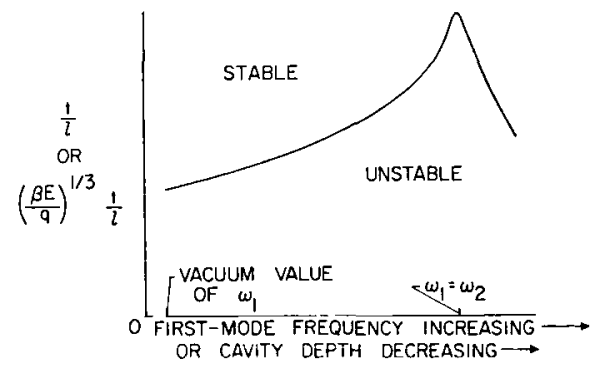

Fig. 5 Qualitative effect on $t / l$ and panel flutter parameter of an increase of the panel first-mode frequency, such as could be caused by a decrease of the cavity depth behind the panel.

would tend to bulge the panel. A panel flutter parameter, involving panel stiffness and stream dynamic pressure, is plotted as a function of panel length-to-width ratio for both clamped-edge and simply supported panels and is compared with the experimental flutter envelope from Ref. 6 . In terms of panel thickness to prevent flutter, the analytical results fall moderately below the experimental envelope, which result is to be expected because the envelope was established largely by the more severe condition of marginal thermal buckling.

The effect of variable Mach number was studied for a clamped-edge aluminum panel at sea level with a length-towidth ratio of 2 . The thickness ratio was essentially constant for $M=1.2,1.3,1.4$, and 1.5 , was $5 \%$ higher at $M=$ 2.0 , and was $6 \%$ lower at $M=1.1$. Another variable Mach number study was made to compare with a series of experiments in a wind tunnel on square and nearly square panels at Mach numbers from 1.16 to 1.45. Analytical results agreed well with the experimental for the thickness ratio but not for the flutter frequency.

The effect of a cavity behind the panel was analyzed qualitatively on the basis of the cavity acting solely as an air spring to resist panel modes that would compress the air, thereby raising those modal frequencies. If the ratio of cavity air density to cavity depth is high enough, the firstmode frequency can be raised to equal or even exceed the second-mode frequency. An equality of first- and secondmode frequencies is generally a very unfavorable factor and tends strongly to produce flutter.

\section{References}

'Stocker, J. E., "A comprehensive review of theoretical and experimental panel flutter investigations," North American Aviation Inc., Columbus Div., Rept. NA61H-444 (September 15,1961 )

${ }_{2}^{2}$ Fung, Y. C. B., "A summary of the theories and experiments on panel flutter," Air Force Office Sci. Res. TN 60-224, Guggenheim Aeronaut. Lab., Calif. Inst. Tech. (May 1960).

${ }^{3}$ Luke, Y. L. and St. John, A., "Supersonic panel Hutter," Wright Air Dev. Center TR 57-252, Midwest Research Inst. (July 1957).

"Zeijdel, E. F. E., "Large deflection panel flutter," Air Force Office Sci. Res. TN 1952, Midwest Research Inst. (January 1962).

'Watkins, C. E., "Three-dimensional supersonic theory," AGARD Manual on Aeroelasticity, edited by W. P. Jones, Vol. 2, Chap. 5 (available through NASA, Washington, D. C.).

"Kordes, E. E., Tuovila, W. J., and Guy, L. D., "Flutter research on skis panels," NASA TN D-451 (1960).

${ }^{7}$ Lock, M. II. and Fung, Y. C., "Comparative experimental and theoretical studies of the flutter of flat panels in a low supersonic flow," Air Force Office Sci. Res. TN 670, Guggenheim Aeronaut. Lab., Calif. Inst. Tech. (May 1961).

${ }^{8}$ Tuovila, W. J. and Presnell, J. G., Jr., "Supersonic panel flutter test results for flat fibre-glass sandwich panels with foamed cores," NASA TN D-827 (1961). 